\title{
Is Gilbert's Syndrome High Prevalence because of Natural Selection; Natural Selection due to Malaria
}

\author{
Adarsh Chauhan* \\ Senior Resident, Department of Pathology, IGMC Shimla, India \\ *Corresponding Author: Adarsh Chauhan, Senior Resident, Department of Pathology, IGMC Shimla, India.
}

Received: June 18, 2019; Published: July 04, 2019

DOI: $10.31080 /$ ASMI.2019.02.0296

\section{Introduction}

GS is characterised by mild unconjugated hyperbilirubinemia, normal hepatic function tests and normal hepatic histology while malaria is a mosquito borne life threatening disease caused by a protozoan parasite. Superficially these two entities appear dissimilar but on closer inspection one can see many similarities between the two. Pathogenesis of both involves liver (decreased hepatic UDP glucuronosyl transferase activity in former while preerythrocytic schizogony in hepatocytes in later). Both have some degree of hemolysis and increased bilirubin levels. Family studies indicate towards independent assortment in GS like that of hereditary hemolytic anemias (which are also malarial natural selection) such as hereditary spherocytosis, G6PD deficiency, and beta thalassemia trait [1]. Geographical distribution of GS is worldwide like that of malaria which has infected people since time immemorial in all the continents except Antarctica.

We hypothesized the connection between the two entities on the basis of present study (a combination of prospective and retrospective study) done at lab at Shimla, capital city of northern Indian Himalayan state of Himachal Pradesh.

\section{Material, Methods and Results}

Liver function tests (biochemical tests) were performed at Dr Lal path labs (EQAS BIORAD certified lab) on Vitrios 350 (Jhonson amd Jhonson make) dry chemistry analyzer during the years 2012 and 2013. These tests were part of preventive health test packages designed for apparently healthy individuals and are routinely done by corporate labs in tie up with health insurance companies. We came across the finding of increased bilirubin up to $2.5 \mathrm{~g} / \mathrm{dl}$ (predominantly unconjugated type) frequently with other hepatic biochemical tests being absolutely normal. Ultrasonography of liver was done (in some cases) which was found to be normal in almost all cases. The prevalence of this isolated benign mild unconjugated hyperbilirubinemia (hence labelled as GS) was found to be about 5 per cent of general population.

\section{Discussion and Conclusion}

These individuals (numbering around 50) were then probed for further clinical history and demographic profile etc. And one thing which stands out common amongst them was their permanent residence or place of birth/ancestry. It so turned out that they happened to be the natives of plains of northern India (Malarial Belt) and were residing in hills due to their job etc. On the contrary we rarely found out this syndrome in individuals who were permanent residents of hills (population living above the so called snowline of malaria). The individuals having GS were then asked whether they had suffered malaria in the past and they replied having no documented history of past illness of malaria.

To conclude we can safely argue that there might be a connection between the above discussed entities and this hypothesis of Gilberts Syndrome being a malarial natural selection (like other natural selection namely sickle cell disease, thalassemias, G6PD deficiency, southeast asian ovalocytosis, elliptocytosis and loss of glycophorin $\mathrm{C}$ and duffy antigen which confer selective avantage adainst malarial infection) [2] needs to be evaluated and tested further.

\section{Acknowledgments}

Dr k l sharma of Dr lal path labs sanjauli shimla hp India.

Volume 2 Issue 8 August 2019

(C) All rights are reserved by Adarsh Chauhan. 